\title{
Menschen binden durch Transparenz
}

\author{
Ulrich Werner
}

Die Gewinnung ehrenamtlichen Engagements ist ein bei der Rechnungslegung sozialer Organisationen in Deutschland vernachlässigter Aspekt, wie eine Analyse deutscher, schweizerischer und englischer Rechnungslegungsnormen zeigt.

Soziale Non-Profit-Organisationen - im Sinne von Organisationen, die etwaige Jahresüberschüsse nicht an die Eigner ausschütten und einen sozial-karitativen Zweck verfolgen - können in unterschiedlichen Rechtsformen (Verein, GmbH, Stiftung etc.) geführt werden. Die Rechtsform, die Kaufmannseigenschaft oder die Größe (vgl. §§ 1 und 5 PublG) einer deutschen Non-Profit-Organisation bestimmen, inwieweit sie ihren Jahresabschluss nach den Vorschriften des Handelsgesetzbuches zu erstellen hat.

So muss eine Non-Profit-Organisation mit der Rechtsform »eingetragener Verein « - vorausgesetzt, der Verein betreibt kein Handelsgewerbe, das nach Art und Umfang einen in kaufmännischer Weise eingerichteten Geschäftsbetrieb erfordert (vgl. §1 HGB) - lediglich wenige aus dem Bürgerlichen Gesetzbuch (BGB) abgeleitete Rechnungslegungsvorschriften beachten (Pflicht zur Rechenschaftslegung nach § 266 BGB in Verbindung mit § 259 f. BGB). Hingegen hat eine als Kapitalgesellschaft geführte NonProfit-Organisation grundsätzlich die Rechnungslegungsvorschriften nach § 264 bis 289 HGB zu befolgen.

Auch wenn eine Non-Profit-Organisation nicht zur Anwendung der Rechnungslegungsnorm nach §§ 264 bis 289 HGB gesetzlich verpflichtet ist, so wird die Beachtung der Norm auf freiwilliger Grundlage empfohlen. Die Aussagekraft der Rechnungslegung ist höher und die Ergebnisse der Unternehmenstätigkeit verschiedener Non-Profit-Organisationen können besser miteinander verglichen werden.

Eine einheitliche, speziell auf Non-Profit-Organisationen ausgerichtete Rechnungslegungsnorm gibt es in Deutschland nicht. Anders in Großbritannien und in der Schweiz: Die schweizerische Norm »Swiss GAAP FER 21 « und britische Norm »Charities SORP 2005 « stellen speziell für Non-Profit-Organisationen entwickelte Rechnungslegungsvorschriften dar.

Dieser Beitrag vergleicht die Rechnungslegungsnorm nach $\S 264$ bis 289 HGB mit Swiss GAAP FER 21 und Charities SORP 2005. Hierbei wird von den Informationsbedürfnissen ehrenamtlicher Mitarbeiter (kurz: Ehrenamtliche) ausgegangen. Schließlich werden Empfehlungen für die Rechnungslegung von Non-Profit-Organisationen in Deutschland skizziert.

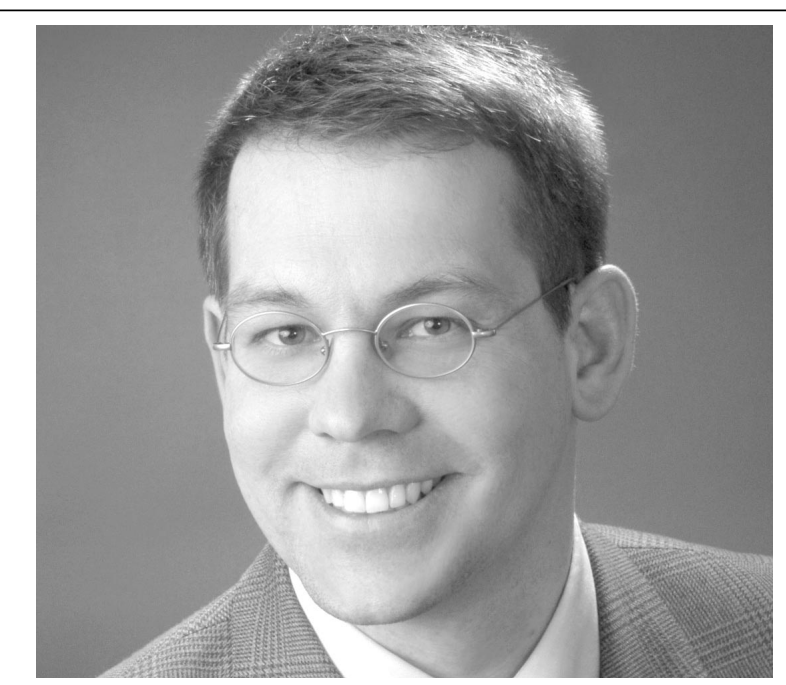

Ulrich Werner (39) studierte Sozialpädagogik an der Berufsakademie Stuttgart, danach Betriebswirtschaft an der Katholischen Universität Eichstätt und der Fernuniversität Hagen. Danach war er in leitenden Positionen in der Altenpflege tätig; seit letztem Jahr ist er bei mehreren Bildungseinrichtungen als Referent für Sozial- und Qualitätsmanagement tätig.

E-Mail: Werner-Amtzell@t-online.de

\section{Inhaltliche Anforderungen an das Rechnungswesen von Non-Profit-Organisationen: der Anspruchsgrup- pen-Ansatz}

Das Umfeld eines Unternehmens kann in unterschiedliche Anspruchsgruppen eingeteilt werden. Es lassen sich einerseits Anspruchsgruppen festmachen, die sowohl für Non-Profit-Organisationen als auch für gewinnorientierte Unternehmen von Bedeutung sind, aber andererseits auch Anspruchsgruppen, die entweder nur den Non-Profit-Organisationen oder nur den gewinnorientierten Unternehmen zuzuordnen sind (vgl. Tabelle 1).

Jede Anspruchsgruppe hat bestimmte Anforderungen an die Rechenschaftslegung von Unternehmen, um anhand der zur Verfügung gestellten Informationen ihre Beziehung zu dem Unternehmen (neu) justieren zu können. So mag ein potenzieller Lieferant an Informationen hinsichtlich der Liquidität des Unternehmens interessiert sein, ein Gewinnteilhaber wird sich für die Ertragslage des Unternehmens interessieren usw. 


\begin{tabular}{|l|c|c|c|}
\hline Anspruchsgruppe & $\begin{array}{c}\text { Sowohl den Non-Pro- } \\
\text { fit-Organisationen als } \\
\text { auch den gewinn- } \\
\text { orientierten Unter- } \\
\text { nehmen zuordnen }\end{array}$ & $\begin{array}{c}\text { Nur den Non-Profit- } \\
\text { Organisationen } \\
\text { zuzuordnen }\end{array}$ & $\begin{array}{c}\text { Nur den gewinnori- } \\
\text { entierten Unterneh- } \\
\text { men zuzuordnen }\end{array}$ \\
\hline Lieferanten & $\mathrm{X}$ & & \\
\hline Kunden, Klienten & $\mathrm{X}$ & & \\
\hline Fremdkapitalgeber & $\mathrm{X}$ & & \\
\hline hauptamtliche Mitarbeiter & $\mathrm{X}$ & $\mathrm{X}$ & \\
\hline ehrenamtliche Mitarbeiter & $\mathrm{X}$ & & \\
\hline Spender & & & \\
\hline Gewinnteilhaber & & & \\
\hline $\begin{array}{l}\text { Allgemeine Öffentlichkeit und weitere } \\
\text { Anspruchsgruppen }\end{array}$ & & & \\
\hline
\end{tabular}

Tabelle 1: Anspruchsgruppen und ihre Informationsbedürfnisse

Im Folgenden soll das Informationsbedürfnis der für Non-Profit-Organisationen typischen Anspruchsgruppe »Ehrenamtliche« genauer untersucht werden. Es soll aufgezeigt werden, welche Anforderungen sich hieraus an die Rechnungslegung ergeben.

Das Thema »Anforderungen an die Rechnungslegung aus der Sicht von Spendern « soll in diesem Beitrag nicht weiter verfolgt werden soll (vgl. Institut der Wirtschaftsprüfer in Deutschland e. V., 1995, S. 415-424).

\section{Das Informationsbedürfnis der Anspruchsgruppe „Ehrenamtliche«}

Welche Informationen beeinflussen den (potenziellen) ehrenamtlichen Mitarbeiter, seine Arbeitskraft einer ganz bestimmten Non-Profit-Organisation zur Verfügung zu stellen? Seine Entscheidung wird im Regelfall von folgenden Faktoren beeinflusst (vgl. Badelt, C., 1999, S. 445 ff.) :

- Wertekonformität: Stimmt die Werteausrichtung der Non-Profit-Organisation mit den Wertevorstellungen des ehrenamtlichen Mitarbeiters überein?

- Art der Tätigkeit: Entspricht die angebotene Art der ehrenamtlichen Tätigkeit den Erwartungen des Ehrenamtlichen?

- Umfang ehrenamtlicher Tätigkeit: Entspricht der angebotene Umfang der ehrenamtlichen Tätigkeit den Erwartungen des Ehrenamtlichen? So mag es beispielsweise von Interesse sein, ob die ehrenamtliche Mitarbeit zeitlich begrenzt ist oder wie hoch die durchschnittliche wöchentliche Arbeitszeit des Ehrenamtlichen ist.

- Nutzen für den Klienten: Welchen Nutzen hat der Hilfebedürftige von der ehrenamtlichen Tätigkeit? Der Nutzen für den Klienten ist insbesondere dann erklärungsbedürftig, wenn der Ehrenamtliche nicht direkt mit dem Klienten zusammenarbeitet, sondern zum Gelingen eines Vor- oder Unterstützungsprozesses beitragen soll.
- Vorteile für den Ehrenamtlichen: Welche Vorteile für den Ehrenamtlichen sind mit seiner freiwilligen Tätigkeit verbunden? Beispiele: Anerkennung, Einfluss, Vergünstigungen, Kontaktmöglichkeiten, Möglichkeiten zum Erwerb zusätzlicher Qualifikationen etc.

- Wirtschaftliche Notwendigkeit ehrenamtlicher Tätigkeit: Ist die Non-Profit-Organisation auf (kostengünstige) ehrenamtliche Mitarbeit angewiesen? Die wirtschaftliche Notwendigkeit ehrenamtlicher Tätigkeit kann danach bemessen werden, wie sich die Ertragslage, die Vermögenslage und die Finanzlage der Non-Profit-Organisation darstellt. Es ist davon auszugehen, dass bei Non-Profit-Organisationen hohe Rücklagen, hohe Jahresüberschüsse, hohe Einzahlungsüberschüsse die Bereitschaft zur ehrenamtlichen Mitarbeit tendenziell eher sinken lassen. Es würde die Notwendigkeit ehrenamtlicher Mitarbeit bezweifelt werden.

- Performanz: Weist die Non-Profit-Organisation eine angemessene Leistungsfähigkeit auf?

Es geht zum einen um die Fragestellung, in welchem Ausmaß die Non-Profit-Organisation effektiv und effizient arbeitet. Während die Effektivität Aufschluss darüber gibt, ob und in welchem Ausmaß die gesetzten Ziele erreicht wurden, beschreibt die Effizienz das Verhältnis von eingesetzten Ressourcen zu erreichten Zielen.

Zum anderen können aus den befolgten Managementund Ethikstandards Rückschlüsse auf die Performanz der Non-Profit-Organisation gezogen wegen. Die Offenlegung von entsprechenden Selbstverpflichtungserklärungen oder die Nennung der erzielten Gütesiegel (mit Verweis auf die dazugehörigen Anforderungskataloge) - beispielsweise DZI - Spendensiegel, ISO 9001-Zertifikat etc. - ermöglichen den Ehrenamtlichen eine bessere Beurteilung des Managements der Non-Profit-Organisation.

Aus den Informationsbedürfnissen der ehrenamtlichen Mitarbeiter können konkrete inhaltliche Anforderungen an die Rechnungslegung abgeleitet werden (vgl. Tabelle 
2). Danach beziehen sich nur wenige Anforderungen auf die quantitative Darstellung der wirtschaftlichen Lage mittels Bilanz, Gewinn- und Verlustrechnung und Kapitalflussrechnung. Vielmehr hat die Rechnungslegung insbesondere auf die qualitativen Informationsbedürfnisse von Ehrenamtlichen einzugehen. Damit steigt bei der Rechnungslegung von Non-Profit-Organisationen die Bedeutung der verbalen Berichterstattung im Verhältnis zu den finanzwirtschaftlichen Tabellenwerken (vgl. Löwe, M., 2003, S. 102 ff.).

\section{Umsetzung der Anforderungen in den Rechnungsle- gungsnormen}

Im Folgenden wird zunächst die Rechnungslegungsnorm nach §§ 264 bis 289 HGB zusammenfassend dargestellt und die darin integrierten Größenerleichterungen beschrieben. Es folgt eine Beurteilung der Norm hinsichtlich der Anforderungen an die Rechnungslegung aus der Sicht von Ehrenamtlichen. Analog wird mit Swiss GAAP FER 21 und mit Charities SORP 2005 verfahren.

Die Rechnungslegungsnorm nach $\S \S 264$ bis 289 HGB verlangt die Erstellung einer Bilanz und einer Gewinnund Verlustrechnung mit jeweils einer weitgehend (vgl. $\S 265$, Abs. $1 \mathrm{HGB}$ ) vorgegebenen Gliederung (\$266 HGB, $\S 275$ HGB). Ferner ist der Jahresabschluss, bestehend aus der Bilanz sowie der Gewinn- und Verlustrechnung, um einen Anhang (§§ 284 ff. HGB) zu erweitern: Hierbei sind allgemeine Angaben zur Bilanzierung und Bewertung, zu einzelnen Positionen der Bilanz und Gewinn- und Verlustrechnung, zu Haftungsverhältnissen und sonstigen finanziellen Verpflichtungen sowie Angaben hinsichtlich der Beziehungen zu verbundenen Unternehmungen, hinsichtlich der Geschäftsführung und des Aufsichtsrates und hinsichtlich der Mitarbeiterzahl zu machen. Die Angabepflichten ergeben sich im wesentlichen aus $\S \S 284 \mathrm{f}$.
HGB sowie aus $\S \S 264,265,268,269,273,274,277,280$, 281, 327 HGB. Rechtsformspezifische Pflichtangaben finden sich zudem im GmbHG, im AktG oder im Abschnitt »Ergänzende Vorschriften für eingetragene Genossenschaften« des Handelsgesetzbuches (vgl. Coenenberg, 2003, S. 860 ff.).

Der Jahresabschluss hat ein tatsächliches Bild der Vermögens-, Finanz- und Ertragslage zu zeichnen. Die Grundsätze ordnungsmäßiger Buchführung sind hierbei zu beachten. Gegebenenfalls sind im Anhang zusätzliche Angaben zu machen, um ein tatsächliches Bild der wirtschaftlichen Lage vermitteln zu können (§ 264, Abs. 2 HGB).

Die Norm sieht auch grundsätzlich die Aufstellung eines Lageberichts (§ $289 \mathrm{HGB}$ ) vor. Der Bericht hat den Geschäftsverlauf, die Lage und das Geschäftsergebnis der Berichtsperiode darzustellen und zu analysieren. Bei der Analyse sind finanzielle Leistungsindikatoren (bei großen Kapitalgesellschaften auch nichtfinanzielle Leistungsindikatoren) mit einzubeziehen. Ferner hat der Lagebericht die voraussichtliche Entwicklung mit ihren wesentlichen Chancen und Risiken zu beurteilen und zu erläutern. Es soll ferner bei der Lageberichterstattung auf das Risikomanagement, auf bedeutende Vorgänge, die nach dem Schluss des Geschäftsjahrs eingetreten sind, auf den Bereich Forschung und Entwicklung sowie auf bestehende Zweigniederlassungen eingegangen werden.

\section{Größenerleichterungen der Rechnungslegungsnorm}

Da die Rechnungslegungsnorm nach §§ 264 bis 289 HGB grundsätzlich die Pflichtnorm für Kapitalgesellschaften darstellt, definiert die Norm in § 267 HGB für Kapitalgesellschaften drei Größenklassen (vgl. Tabelle 3). Für eine Einstufung müssen mindestens zwei der drei Kriterien an zwei aufeinander folgenden Abschlussstichtagen erfüllt sein.

\begin{tabular}{|ll|}
\hline Informationsbedürfnis & Anforderung an die Rechenschaftslegung \\
\hline Wertekonformität & $\begin{array}{l}\text { Beschreibung der Unternehmensphilosophie, des Leitbildes, der Werteausrichtung oder } \\
\text { Ähnliches. }\end{array}$ \\
\hline Urt der Tätigkeit & $\begin{array}{l}\text { Beschreibung der Betätigungsfelder der Non-Profit-Organisation im Allgemeinen; Be- } \\
\text { schreibung der Tätigkeiten, die von Ehrenamtlichen typischerweise ausgeführt werden. }\end{array}$ \\
\hline Nutzen für den Klienten & $\begin{array}{l}\text { Quantifizierung der geleisteten ehrenamtlichen Tätigkeit und der durchschnittlichen } \\
\text { Anzahl der Ehrenamtlichen; Beschreibung typischer Arbeitszeitmodelle für Ehrenamtli- } \\
\text { che. }\end{array}$ \\
\hline Vorteile für den Ehrenamtlichen & $\begin{array}{l}\text { Beschreibung, welchen Nutzen die Klienten durch die ehrenamtliche Tätigkeit haben. } \\
\text { Arbeit. }\end{array}$ \\
\hline $\begin{array}{l}\text { Wirtschaftliche Notwendigkeit } \\
\text { ehrenamtlicher Tätigkeit }\end{array}$ & $\begin{array}{l}\text { Darstellung der Vermögens-, Ertrags- sowie Finanzlage in Form einer Bilanz, } \\
\text { Gewinn- und Verlustrechnung sowie Kapitalflussrechnung; Beschreibung der wirtschaft- } \\
\text { lichen Lage im Berichtszeitraum; Darstellung der erwarteten wirtschaftlichen Lage mit }\end{array}$ \\
\hline Erörterung etwaiger Chancen und Risiken. \\
\hline $\begin{array}{l}\text { Beschreibung nachprüfbarer Ziele und Beschreibung des Zielerreichungsgrades von in } \\
\text { Vorperioden festgelegten Zielsetzungen; Beschreibung und Quantifizierung des Auf- } \\
\text { wandes zur Erreichung der Zielsetzungen; Darstellung der erhaltenen Gütesiegel mit } \\
\text { Verweis auf die dazugehörigen Anforderungskriterien; Verweis auf offen gelegte Selbst- } \\
\text { verpflichtungserklärungen. }\end{array}$ \\
\hline
\end{tabular}

Tabelle 2: Anforderungen an die Rechnungslegung ais der Sicht ehrenamtlicher Mitarbeiterinnen und Mitarbeiter 


\begin{tabular}{|c|c|c|c|}
\hline & \multicolumn{3}{|c|}{ Kriterien } \\
\hline Größenklasse & Bilanzsumme & Umsatzerlöse & Arbeitnehmer \\
\hline $\begin{array}{l}\text { Kleine Kapital- } \\
\text { gesellschaft }\end{array}$ & $\leq 4.015$ TEUR & $\leq 8.030$ TEUR & $\leq 50$ \\
\hline $\begin{array}{l}\text { Mittelgroße } \\
\text { Kapitalgesell- } \\
\text { schaft }\end{array}$ & $\begin{array}{c}>4.015 \text { und } \\
\leq 16.060 \\
\text { TEUR }\end{array}$ & $\begin{array}{c}\leq 8.030 \text { und } \\
\leq 32.120 \\
\text { TEUR }\end{array}$ & $\begin{array}{c}>50 \text { und } \\
\leq 250\end{array}$ \\
\hline $\begin{array}{l}\text { Große Kapi- } \\
\text { talgesellschaft }\end{array}$ & $\begin{array}{c}>16.060 \\
\text { TEUR }\end{array}$ & $\begin{array}{c}>32.120 \\
\text { TEUR }\end{array}$ & $>250$ \\
\hline
\end{tabular}

Tabelle 3: Die drei Größenklassen des Handelgesetzbuches und ihre Kriterien $($ TEUR $=$ Tausend Euro; TCFR = tausend Schweizer Franken)

\begin{tabular}{|l|c|c|c|}
\hline & \multicolumn{3}{|c|}{ Kriterien } \\
\hline Größenklasse & Bilanzsumme & $\begin{array}{c}\text { Erlöse aus un- } \\
\text { entgeltlichen } \\
\text { Zuwendun- } \\
\text { gen und öf- } \\
\text { fentlichen } \\
\text { Beiträgen }\end{array}$ & $\begin{array}{c}\text { Arbeitneh- } \\
\text { mer-Stellen }\end{array}$ \\
\hline $\begin{array}{l}\text { Kleine } \\
\text { Non-Profit- }\end{array}$ & $\begin{array}{c}\leq 2.000 \text { TCFR } \\
\text { Organisation }\end{array}$ & $\begin{array}{c}\leq 1.000 \text { TCFR } \\
(\approx 646 \text { TEUR })\end{array}$ & $<10$ \\
\hline $\begin{array}{l}\text { Große } \\
\text { Non-Profit- } \\
\text { Organisation }\end{array}$ & $\begin{array}{c}(\approx 2.000 \text { TCFR } \\
(\approx 1.292\end{array}$ & $>1.000$ TCFR & $>10$ \\
& & $\approx 646$ TEUR $)$ & \\
\hline
\end{tabular}

Tabelle 4: Die zwei Größenklassen der Schweizer Rechnungslegungsnorm und ihre Kriterien (TEUR = Tausend Euro; TCFR = tausend Schweizer Franken)

Kleine Kapitalgesellschaften können eine verkürzte Bilanz mit einer weniger tief gehenden Aufgliederung der übergeordneten Positionen aufstellen (§ 266, Abs. 1 HGB). Sie können bei der Gewinn- und Verlustrechnung bestimmte Einzelposten zusammenfassen (§ 276 HGB).

Kleine und mittelgroße Kapitalgesellschaften haben weniger Einzelangaben im Anhang zu machen. Kleine Kapitalgesellschaften sind von der Pflicht zur Aufstellung eines Lageberichtes befreit. Ferner hängen die Fristen zur Aufstellung der Jahresabschlüsse von den Größenklassen ab.

\section{Beurteilung der Rechnungslegungsnorm}

Die Norm ist grundsätzlich geeignet, sich einen umfassenden Überblick über die wirtschaftliche Lage der NonProfit-Organisation zu verschaffen. Hieraus können dann Schlüsse gezogen werden, ob die Non-Profit-Organisation auf ehrenamtliche Mitarbeiter (in wirtschaftlicher Hinsicht) angewiesen ist. So informieren die Bilanz und die Gewinn- und Verlustrechnung grundlegend über die Vermögens- und Ertragslage des Unternehmens. Eine detaillierte zahlenmäßige Darstellung der Finanzlage in Form einer Kapitalflussrechnung ist nicht vorgeschrieben. Gerade die Verbindung zwischen dem vergangenheitsorientierten Jahresabschluss und der eher zukunftsorientierten Lageberichterstattung ermöglicht dem Adressat der Rechnungslegung, sich einen umfassenden Überblick über die aktuelle und erwartete wirtschaftliche Lage der Non-Profit-Organisation $\mathrm{zu}$ verschaffen.

Eine Darstellung der von der Non-Profit-Organisation verfolgten Ziele, der Zielerreichungsgrade und der Maßnahmen verlangt die HGB-Norm nicht. »Insbesondere Informationen über die Sachzielerreichung sind [...] nicht vorgesehen « (Löwe, M., 2003, S. 304). Auf »Ziele und Strategien « muss im Lagebericht nicht eingegangen werden (vgl. Kaiser, K., 2005, S. 407 ff.). Rückschlüsse auf die Performanz der Non-Profit-Organisation können somit nicht oder nur sehr eingeschränkt gezogen werden. Die Rechnungslegungsnorm nach $\S 264$ bis 289 HGB geht auch auf andere Informationsbedürfnisse von Ehrenamtlichen, abgesehen von den Informationen über die wirtschaftliche Lage, nicht (direkt) ein. Da die HGB-Norm schwerpunktmäßig die finanziellen Aspekte des Unternehmens beleuchtet und somit insbesondere das Informationsbedürfnis der Gläubiger und der Anteilseigner fokussiert, verwundert das Ergebnis allerdings nicht.

Angesichts der Bedeutung eines Lageberichtes zur Beurteilung qualitativer Merkmale einer Non-Profit-Organisation wäre die Erstellung eines Lageberichts aus der Sicht von Ehrenamtlichen auf jeden Fall angebracht - selbst dann, wenn die rechnungslegende Non-Profit-Organisation als klein einzustufen ist.

\section{Die Schweizer Norm Swiss GAAP FER 21}

Die Rechnungslegungsnormenreihe Swiss GAAP FER wird herausgegeben von der schweizerischen »Stiftung für Fachempfehlungen zur Rechnungslegung «. »GAAP« steht für »General accepted accounting principles « und »FER « für Fachempfehlung zur Rechnungslegung (vgl. Stiftung für Fachempfehlungen zur Rechnungslegung, 2005, S. 147 ff.; Internet http://www.fer.ch). Die Fachempfehlung mit der Ordnungsnummer 21 - kurz: »Swiss GAAP FER 21 - normiert die Rechnungslegung für gemeinnützige, soziale Non-Profit-Organisationen.

Eine gesetzliche Verpflichtung zur Anwendung der Swiss GAAP FER 21 besteht für schweizerische Non-ProfitOrganisationen nicht. Allerdings ist die Umsetzung der Swiss GAAP FER 21 eine Voraussetzung zur Erlangung des schweizerische Spendengütesiegel »ZEWO-Gütesiegel « (Internet http://www.zewo.ch). Die Norm Swiss GAAP FER 21 wurde im November 2002 in Kraft gesetzt.

Swiss GAAP FER 21 besteht aus sechs Abschnitten: 1 . Präambel, 2. Definitionen, 3. Empfehlungen, 4. Erläuterungen (mit ergänzenden Anmerkungen zu den Empfehlungen), 5. Anhänge (mit konkreten Umsetzungsbeispielen) und 6. Glossar (Begriffsdefinitionen).

In der Präambel wird auf die Zielsetzung von Swiss GAAP FER 21 eingegangen. Im Abschnitt Definitionen werden der Begriff "gemeinnützige, soziale Non-Profit-Organisation « erläutert sowie große und kleine Non-ProfitOrganisationen von einander abgegrenzt.

Der Abschnitt Empfehlungen stellt das Kernstück der Swiss GAAP FER 21 dar: Darin wird zum einen auf die anzuwendenden »Grundlagen und Grundsätze« der Rechnungslegung eingegangen. Hierbei handelt es sich um 
eine Zusammenstellung der üblichen Grundsätze ordnungsmäßiger Buchführung. Zum anderen werden die Bestandteile des Jahresabschlusses nach Swiss GAAP FER 21 erläutert; dazu zählen:

- Bilanz: Der Aufbau der Bilanz orientiert sich zwar grundsätzlich an den Vorgaben für gewinnorientierte Unternehmen. Dennoch sind einige Gliederungspositionen spezifisch für Non-Profit-Organisationen: Auf der Passivseite sind die kurzfristigen und langfristigen Verbindlichkeiten, das Organisationskapital (im Sinne von »Eigenkapital « der Non-Profit-Organisation) und die zweckbestimmten (Spenden)-Fonds auszuweisen. Frei verfügbare (Spenden)-Fonds werden dem Organisationskapital zugerechnet.

- Betriebsrechnung: Die Betriebsrechnung ähnelt einer Gewinn- und Verlustrechnung kommerzieller Unternehmen. Die Betriebsrechnung kann sowohl nach dem Gesamtkostenverfahren als auch nach dem Umsatzkostenverfahren erstellt werden. Verwaltungsaufwendungen müssen gesondert ausgewiesen werden, ebenso müssen die Veränderungen der zweckgebundenen Fonds gesondert und brutto ausgewiesen werden.

- Mittelflussrechnung: Die Mittelflussrechnung ist eine Kapitalflussrechnung mit differenziertem Ausweis des Kapitalflusses aus Betriebstätigkeit, aus Investitionstätigkeit und aus Finanzierungstätigkeit.

- Rechnung über die Veränderung des Kapitals: Die Rechnung über die Veränderung des Kapitals stellt in einer Tabelle die Zuweisung, die Verwendung und die Anfangs- und Endbestände des Organisationskapitals einerseits und der (zweckbestimmten) Fonds andererseits dar.

- Anhang: Der Anhang enthält die angewandten Bilanzierungs- und Bewertungsgrundsätze und Erläuterungen zu den einzelnen Positionen der Tabellenwerke.

- Leistungsbericht: Der Leistungsbericht hat in angemessener Art die Effektivität und Effizienz der Non-ProfitOrganisation zu beschreiben. Der Leistungsbericht muss auf den Zweck der Organisation eingehen, Auskunft über die leitenden Organe und ihre Amtszeit geben, die für die Geschäftsführung verantwortlichen Personen sowie die Verbindungen zu nahe stehenden Organisationen offen legen. Ferner legt der Leistungsbericht »zwingend offen [...] die gesetzten Ziele und eine Beschreibung der erbrachten Leistungen in Bezug auf die gesetzten Ziele und die Verwendung der zur Verfügung stehenden Mittel« (Randziffern 41 f. Swiss GAAP FER 21).

In dem Abschnitt Erläuterungen der Swiss GAAP FER 21 wird empfohlen, in den Leistungsbericht noch Angaben zu folgenden Themen aufzunehmen: Beurteilung der $\mathrm{Zu}$ friedenheit der Leistungsempfänger oder Begünstigten, Beschreibung der geplanten Leistungen, Angaben darüber, wie das Erreichen qualitativer Ziele gemessen und beurteilt werden kann, aussagekräftige Kennzahlen für die Erreichung der gesetzten Ziele sowie Hauptrisiken und anfällige Systeme (Randziffer 58 Swiss GAAP FER 21).

Die Swiss GAAP FER 21 unterscheidet lediglich zwischen zwei Größenklassen (vgl. Tabelle 4, Seite 27).

Kleine Non-Profit-Organisationen müssen gemäß Swiss GAAP FER 21 keine Mittelflussrechnung erstellen und sind nicht verpflichtet, Erträge und Aufwendungen periodengerecht abzugrenzen.

\section{Beurteilung der Swiss GAAP FER 21}

Die Umsetzung der Norm trägt dazu bei, die wirtschaftliche Lage der Non-Profit-Organisation umfassend darzustellen. Die Aufstellung einer Kapitalflussrechnung ermöglicht dem Ehrenamtlichen, sich ein genaueres Bild auch über Finanzlage der Non-Profit-Organisation zu machen. Anhand des Leistungsberichtes können Schlussfolgerungen hinsichtlich der Performanz der Non-ProfitOrganisation gezogen werden. Auf abgegebene Selbstverpflichtungserklärungen oder erhaltene Gütelabels ist nicht näher einzugehen.

Auf eine Beschreibung der Arten der ehrenamtlichen Mitwirkungsmöglichkeiten verzichtet die Norm. Positiv ist, dass bei Anwendung der Norm der Rechnungslegung entnommen kann, in welchem Ausmaß freiwillige Arbeit stattgefunden hat. Eine Darstellung der gewährten Anreize für ehrenamtliche Mitarbeit sieht die Norm nicht vor.

\section{Die britischen Charities SORP 2005}

»Charities SORP 2005« stellt eine Abkürzung für die Rechnungslegungsnorm »Accounting and Reporting by Charities: Statement of Recommended Practice (revised 2005)« dar. Die Charities SORP 2005 gilt in Großbritannien als die allgemein gültige Grundlage für das externe Rechnungswesen von Non-Profit-Organisationen.

Die Norm erfüllt grundsätzlich die gesetzlichen Anforderungen an das externe Rechnungswesen von Non-Profit-Organisationen und entspricht auch den grundsätzlichen Rechungswesen-Anforderungen des britischen Standardisierungsrates »Accounting Standards board (vgl. Dawes, G., 1994, S. 88 ff.). Herausgeben werden die Charites SORP 2005 von der »Charity Comission« für England und Wales. Die Charity Comission ist eine gesetzlich verankerte Institution, mit der Aufgabe, Wohlfahrtsorganisationen zu registrieren und ihre Arbeit zu regulieren (vgl. Internet http://www.charity-comission.gov.uk, Dawes G., 1994, S. 75 ff. und Löwe, M., 2003, S. 169 ff.).

Nach dem Einführungsabschnitt werden die einzelnen Bestandteile der Rechnungslegung für Charities kapitelweise behandelt. Den Themen »Consolidation of Subsidary Undertakings«, »Associates, Joint Ventures and Joint Arrangements «, »The SORP in relation to Charitable Companies in the UK« und »Accounting for Retirement Benefits« werden eigene Kapitel gewidmet.

Die Charities SORP 2005 ist mit 103 DIN-A4 Seiten deutlich umfangreicher als die Swiss GAAP FER 21. Bereits in dem Einführungskapitel werden die Elemente der Rechnungslegung von Charities umschrieben. Diese sind:

- Ergebnisrechnung (Statement of Financial Activities): Hierbei handelt es sich um eine tabellarische Zusammenstellung der Einkünfte und Aufwendungen gegliedert nach frei verfügbaren Fonds, beschränkt verfügbaren Fonds und den »Endowment Funds« (im Sinne von Dotationsmittel, Stiftungskapital). Die Erklärung über die finanziellen Aktivitäten ist in seiner Grundstruktur mit einer Gewinn- und Verlustrechnung vergleichbar.

- Bilanz (Balance sheet): Die Bilanz vermittelt einen Überblick über das Anlage- und Umlaufvermögen, über 
die kurz- und langfristigen Verbindlichkeiten und über die Bestände der einzelnen Fonds.

- Kapitalflussrechnung (Cash flow statement)

- Anhang (notes): Der Anhang erläutert die angewendeten Rechnungslegungsgrundsätze und geht auf einzelne Position der Ergebnisrechnung, der Bilanz und der Kapitalflussrechung genauer ein.

- Jahresbericht (Trustees' Annual Report): Der Jahresbericht hat die Aufgabe jene Themenbereiche zu erläutern, die sich in Zahlenwerken nur unbefriedigend darstellen lassen. Der Bericht soll sowohl den gesetzlichen Anforderungen genügen als auch einen Überblick über die Fern- und Nahziele, die Maßnahmen und die Performance liefern. Der Trustees' Annual Report informiert über Jahresziele, Planungen für die Zukunft, über das Governance über die Leitungsstruktur (vgl. Randziffer 36 Charities Sorp 2005).

Die geforderten Inhalte des Jahresberichtes sollen im Folgenden zusammenfassend dargestellt werden:

- »Reference and Administrative Details of the Charity, its Trustees and Advisors «: Zu den administrativen Angaben gehören unter anderem Name und Adresse der Non-Profit-Organisation, Namen der Geschäftsführer sowie Namen und Adressen von für die Non-Profit-Organisation relevanten Personen und Organisationen wie Auditoren, Berater etc. (Randziffern $41 \mathrm{ff}$. Charities SORP 2005).

- »Structure, Governance and Management«: Hier soll verständlich gemacht werden, wie die Entscheidungsprozesse innerhalb der Non-Profit-Organisation geregelt sind. $\mathrm{Zu}$ erläutern sind die Gründungsdokumente und die rechtliche Verfassung der Non-Profit-Organisation, die Methoden zur Gewinnung, Ernennung, Einführung und Einarbeitung der Verwalter, die Verbindungen zu einem größeren Netzwerk sowie die Beziehungen zwischen der Charity und den mit ihr verbundenen Gruppen. Auch sollte darauf eingegangen werden, wie mit den wichtigsten Risiken umgegangen wird (Randziffern 44 ff. Charities SORP 2005).

- »Objectives and Activities «: Dem Leser sind die von der Non-Profit-Organisation gesetzten Ziele und Maßnahmen verständlich zu machen. Beschrieben werden sollen unter anderem die Jahreshauptziele und die Strategien, wie die anvisierten Ziele zu erreichen sind (Randziffern 47 ff. Charities SORP 2005). Unter anderem ist auf die Ziele und auf die Maßnahmen im Hinblick auf ehrenamtliche Tätigkeiten näher einzugehen: So sei es für den Leser des Trustee's Annual Report wichtig, über die Rolle und den Beitrag der Ehrenamtlichen informiert zu werden. Dies könne in der Form geschehen, dass Maßnahmen, bei deren Umsetzung Ehrenamtliche mitwirken, beschrieben werden. Die von Ehrenamtlichen geleistete Arbeit kann durch Stundenangaben oder durch umgerechnete Vollzeitäquivalente quantifiziert werden. Hieraus kann ein (ökonomischer) Wert der Freiwilligen-Arbeit abgeleitet werden (vgl. Randziffer 51 Charities SORP 2005).

- »Achievements and Performance«: Es sollen Informationen über die erreichten Ziele bereitgestellt werden. Ein Überblick über die Performanz hinsichtlich der gesetzten Ziele soll vermittelt werden. Die Maßstäbe und Indikatoren, an Hand derer der Zielerreichungsgrad gemessen werden kann, sollen dargestellt werden (Randziffern 53 f. Charities SORP 2005).
- »Plans for Future Periods«: Die Planungen für zukünftige Perioden - die Zielsetzungen und die geplanten Maßnahmen zur Zielerreichung - sind aufzuzeigen (Randziffern 57 f. Charities SORP 2005).

- »Financial Review«: Die finanzielle Lage und die angewendete Finanzstrategie sind zu erläutern (Randziffern 55 f. Charities SORP 2005).

- »Funds held as Custodian Trustee on Behalf of Others «: Verwaltet eine Charity stellvertretend auch das Vermögen einer anderen Charity, so ist hierauf mit diversen Angaben näher einzugehen (Randziffer 59 Charities SORP 2005).

Eine Charity mit einem Bruttojahreseinkommen bis 100.000 Britische Pfund (145.550 Euro), sofern sie nicht eine Gesellschaft im Sinne des Companies Act 1985 ist, muss Erträge und Aufwendungen nicht periodengerecht abgrenzen.

Charities aus England oder Wales mit einem Bruttojahreseinkommen oder Jahresausgaben bis 250.000 Britische

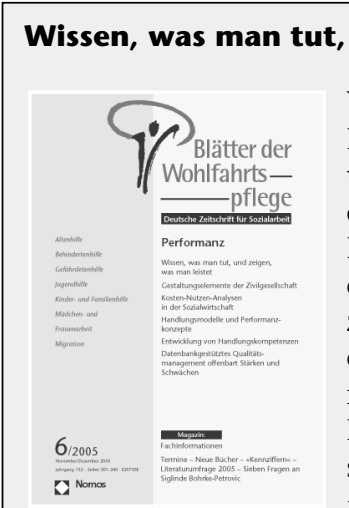

Welchen Nutzen stiften soziale Dienste und Einrichtungen? Was tut Soziale Arbeit und wie stellt sie das, was sie tut, nach außen dar? Darum geht es bei der Frage nach der Performanz professioneller Sozialarbeit. Es geht also sowohl um die Leistung selbst als auch um deren Darstellung. Industriebetriebe können den Ausstoß ihrer Produkte zählen und »das Ergebnis nach Steuern« an die Anteilseigner abführen. Das Erkennen und die Vermittlung dessen, was im Alltag sozialer Dienste und Einrichtungen geleistet wird, ist schwieriger zu erfassen und zu vermitteln. Dennoch ist es hohe Zeit, dass sich Wissenschaft und Praxis Sozialer Arbeit dieser Aufgabe zuwenden. Der Umbau des Sozialstaates, die Krise der öffentlichen Leistungsträger, das wachsende Misstrauen der Öffentlichkeit und nicht zuletzt das Interesse der Mitarbeiterschaft und der Nutzer selbst lassen keine Wahl, als sich der Gretchenfrage zu stellen: Wem bieten wir welchen Nutzen unter Einsatz welcher Ressourcen? Eine ausschließlich wirtschaftliche Rechenschaftslegung wird die gesellschaftliche Bedeutung Sozialer Arbeit nicht erfassen können, wenngleich die ordnungsgemäße Bilanz unabdingbar zur Performanz sozialer Dienste und Einrichtungen gehört. Dazu treten müssen Informationen über die Erreichung von Sachzielen und die Offenlegung der aufgewendeten Mittel im Vergleich zum entstandenen Nutzen. Soziale Organisationen müssen, so die Blätter der Wohlfahrtspflege, im eigenen Interesse und aus Verpflichtung gegenüber der Gesellschaft nachweisen, dass aus den begrenzten Ressourcen ein Maximum an Effektivität (»die richtigen Dinge tun«) und Effizienz (»die Dinge richtig tun «) erreicht wurde. Die Beiträge im neuen Themenheft der Blätter der Wohlfahrtspflege zeigen, wie dies in Theorie und Praxis aussehen kann.

Das Einzelheft 6/2005 der Blätter der Wohlfahrtspflege kostet 10,- Euro; das Jahresabonnement mit sechs Ausgaben 55 ,- Euro.

Nomos Verlagsgesellschaft, 76520 Baden-Baden,

Telefon 07221 2104-39, Fax 07221 210443,

E-Mail hohmann@nomos.de 
Pfund (363.875 Euro) haben zwar einen Trustees' Annual Report zu erstellen, der sich eng an dem Trustees' Annual Report einer großen Charity orientiert; sie sind allerdings von einzelnen Angabepflichten befreit (Charities SORP 2005, Tabelle 11). Unter anderem muss nicht auf die Planungen für zukünftige Perioden eingegangen werden. Auch kann auf eine explizite Erläuterung des Beitrages von Ehrenamtlichen in der Non-Profit-Organisation verzichtet werden.

\section{Beurteilung der Charities SORP 2005}

Die Charities SORP 2005 decken wichtige Anforderungen an die Rechnungslegung aus Sicht der Ehrenamtlichen ab. Durch die vorgeschriebenen Erläuterungen hinsichtlich der Gründungsdokumente sind Rückschlüsse auf die Wertevorstellungen der Non-Profit-Organisation möglich. Positiv ist zu bewerten, dass - zumindest bei größeren Charities - die Rolle und die Leistungen der Ehrenamtlichen zu beschreiben ist. Die Stärke der Norm liegt insbesondere darin, dass Rückschlüsse auf die Performanz der Non-Profit-Organisation gezogen werden können, indem auf die Ziele und auf Strategien, also auf Aktivitäten zur Zielerreichung einzugehen ist. Eine Darstellung der gewährten Anreize bei ehrenamtlicher Mitarbeit sieht allerdings die Norm ebenfalls nicht vor.

\section{Empfehlungen für die Rechnungslegung deutscher Non-Profit-Organisationen}

Welche Konsequenzen können sich aus der unterschiedlichen Beurteilung der analysierten Rechnungslegungsnormen für deutsche Non-Profit-Organisationen ergeben? Drei Strategievarianten sind denkbar.

Variante 1: Anwendung der Rechnungslegungsnorm nach $\S \S 264$ bis 289 HGB mit Integration weitergehender Angaben in den Anhang und insbesondere in den Lagebericht.

Variante 2: Anwendung der Rechnungslegungsnorm nach $\S \S 264$ bis 289 HGB und Aufstellung eines zweiten Abschlusses gemäß einer - aus Sicht der Ehrenamtlichen -

\section{Literatur}

Badelt, C.: Ehrenamtliche Arbeit im Nonprofit Sektor, in Badelt, C., 1999, S. 433-462.

Badelt, C.: Handbuch der Nonprofit-Organisation, Stuttgart 1999.

Coenenberg, A.: Jahresabschluss und Jahresabschlussanalyse, Stuttgart 2003.

Greyham, D.: Rechnungslegung in England und Wales, in: Walz, W.R., 1994, S. 75-117.

Institut der Wirtschaftsprüfer in Deutschland e. V.: HFA Stellungnahme 4/1995: Zur Rechnungslegung und Prüfung spendensammelnder Organisationen, in: IDW-Fachnachrichten 1995, S. $415-425$.

Kaiser, K.: Auswirkungen des Bilanzreformgesetzes auf die zukunftsorientierte Lageberichterstattung, in: Die Wirtschaftsprüfung 2005, Heft 8, S. 405-418.

Löwe, M.: Rechnungslegung von Non-Profit-Organisationen, Berlin 2003.

Stiftung für Fachempfehlungen zur Rechnungslegung: Swiss GAAP FER 2005/06, Zürich 2005

Walz, W. R.: Rechnungslegung und Transparenz im Dritten Sektor, Köln 2004. besser geeigneten Rechnungslegungsnorm (z. B. Charities SORP 2005 bzw. Swiss GAAP FER 21).

Variante 3: Rechnungslegung ausschließlich nach Swiss GAAP FER 21 bzw. Charities SORP 2005.

Gegen die Variante 2 dürfte der hohe Aufwand sprechen, der mit der Erstellung zweier Rechnungslegungen verbunden ist. Gegen Variante 3 spricht die Tatsache, dass ein Vergleich von Non-Profit-Organisationen, die die HGB-Rechnungslegungsvorschriften anwenden oder anwenden müssen, mit jenen, die ausschließlich nach Swiss GAAP FER 21 bzw. Charities SORP 2005 Rechnung legen, erschwert wird.

Bei Variante 1 hingegen könnten die Angaben, die den Anforderungen an die Rechnungslegung aus der Sicht der Ehrenamtlichen genügen, bei der Rechnungslegung Berücksichtigung finden. Die weite Verbreitung der Rechnungslegung gem. §§ 264 ff. HGB stellt die Vergleichbarkeit von Jahresabschlüssen auf eine breite Basis (vgl. Löwe M., 2003, S. 305 f.).

Bei der Umsetzung von Variante 1 sollten folgende Punkte beachtet werden: Die Integration einer Kapitalsflussrechnung in die Rechnungslegung von Non-ProfitOrganisationen wird empfohlen. Auf die Werteausrichtung der Non-Profit-Organisation sollte beispielsweise im Lagebericht eingegangen werden. Im Lagebericht sind Angaben zur Effektivität und Effizienz der Non-Profit-Organisation in Anlehnung an die Vorschriften zum Trustees' Annual Report in den Charities SORP 2005 zu machen. Das setzt eine freiwillige Beschreibung der für die Folgeperiode(n) festgelegten Ziele und der geplanten Maßnahmen zur Zielerreichung (»Strategien «) voraus. In die Lageberichtserstattung sollte der Erreichungsgrad der in der Vergangenheit gesetzten Ziele aufgezeigt werden. Einzugehen ist auf erreichte Gütelabels und auf Selbstverpflichtungserklärungen.

Eine Darstellung, wie durch ehrenamtliche Tätigkeit der Hilfszweck der Non-Profit-Organisation besser erfüllt werden kann und welche Möglichkeiten ehrenamtlichen Engagements hinsichtlich Art und Umfang bestehen, ist sinnvoll. Die Arbeitsleistung durch ehrenamtliche Mitarbeit im Berichtsjahr und die Anzahl der Ehrenamtlichen sollte quantifiziert werden. Die Anreize für ehrenamtliche Tätigkeit sollten dargestellt werden.

Auch bei der verbalen Berichterstattung ist auf Vollständigkeit, Richtigkeit und Klarheit zu achten. Wird hingegen nur über die positiven Entwicklungen berichtet, obwohl gewisse Defizite (im Hinblick auf die anvisierten Ziele) zu verzeichnen waren, so würde das diesen Grundsätzen widersprechen.

Um eine periodenübergreifende Vergleichbarkeit zu ermöglichen, ist zudem auf Kontinuität bei der Berichterstattung zu achten. Bei Zahlenangaben (Anzahl ehrenamtlicher Mitarbeiter, Arbeitsvolumen der ehrenamtlichen Tätigkeit in Stunden usw.) ist dem Jahreswert zumindest der Vorjahreswert gegenüberzustellen.

Die Rechenschaftslegung sollte - auch wenn eine gesetzliche Verpflichtung hierzu nicht besteht - stets vor ihrer Veröffentlichung von einem Unabhängigen geprüft werden. 